\title{
NEW RECORDS OF THE GENUS Iotonchus (Mononchida, Iotonchidae) FOR VIETNAM FAUNA AND AN UPDATED KEY TO SPECIES FROM VIETNAM
}

\author{
Vu Thi Thanh Tam \\ Institute of Ecology and Biological Resources, VAST, vtam7572@yahoo.com
}

\begin{abstract}
Five species of the genus Iotonchus (Cobb, 1916) Altherr, 1950, viz. Iotonchus arcuatus, I. candelabri, I. paracutus, I. pusillus and I. singaporensis, were recorded for the first time in Vietnam. All newly recorded species were also redescribed and illustrated, these species are recorded from Cat $\mathrm{Ba}$ and Bidoup-Nui Ba National Parks. In addition, the current status of two species, I. helicus and I. thui, which were originally discovered in Vietnam was discussed. An updated key to all extant species from Vietnam is also provided.
\end{abstract}

Keywords: Mononchida, Iotonchidae, Iotonchus, key, new record, Vietnam.

\section{INTRODUCTION}

In Vietnam up to now, studies on predaceous nematodes of the order Mononchida identified 15 species of the genus Iotonchus (Iotonchidae) could be found in a few of works $[8,10]$. In the present paper, five of the genus Iotonchus species, I. arcuatus Khan, Araki \& Bilgrami, 2000, I. candelabri Yeates, 1992, I. paracutus Vinciguerra \& Orselli, 2000, I. pusillus Loof, 2006 and I. singaporensis Ahmad, Baniyamuddin \& Jairajpuri, 2006, were identified and represent new records for the nematode fauna in Vietnam, all species were collected in Cat $\mathrm{Ba}$ and BidoupNui Ba National Parks.

Due to confliction of some important characters between description and figures in the original article of I. helicus and I. thui [9], we decided to check the hoplotype and paratypes, which were deposited and preserved at the Department of Nematology, IEBR (Dr. Nguyen Vu Thanh's collection). Unfortunately, all type material including of holotype, paratypes and syntypes of I. helicus Nguyen, 2006 and I. thui Nguyen, 2006 were not available. The validity of these species is discussed.

\section{MATERIALS AND METHODS}

Soil samples were collected from pristine tropical forest areas in Cat $\mathrm{Ba}$ and Bidoup-Nui Ba National Parks (Vietnam). Nematodes were extracted from soil sample by modified
Baermann funnel technique [12], killed by heat, fixed in TAF solution ( 7 formalin 40\%: 2 triethanolamine: 91 distilled water), transferred to anhydrous glycerol according to Seinhorst (1959) [11], and mounted on glass slides for study. Specimens were drawn using an Olympus microscope $\mathrm{CH} 40$ with drawing tube and illustrations were edited by Adobe Illustrator CS.

\section{RESULTS AND DISCUSSION}

\section{Iotonchus arcuatus Khan, Araki \& Bilgrami, 2000 (Fig. 1)}

Material examined: 18 females in good condition. Measurements: see table 1.

Female: Moderately slender nematodes of medium size, 1.3-1.6 mm long. Habitus after fixation ventrally arcuate, especially towards posterior end. Cuticle smooth, 3-4 m thick throughout body. Lip region slightly offset from body contour by depression, 36-39 m width and 12-15 $\mathrm{m}$ height. Labial papillae prominent, protruded. Amphidial fovea cup shaped, aperture situated at 14-16 m from anterior end of body. Buccal cavity medium size 27-34 m length and $15-22 \mathrm{~m}$ width or about 1.5 times as long as wide, strongly sclerotized. Dorsal tooth with sharp apex, pointing forward, located at 9-10 m or $22-25 \%$ of buccal cavity length from its base. Oesophagus cylindroid, 279-320 m long, nerve ring at about one quarter of its length from anterior end; secretory-excretory pore located 
just posterior to nerve ring. Oesophago-intestinal junction tuberculate. Intestine with wide lumen. Reproductive system didelphic-amphidelphic, both branches equally developed with ovary reflexed. Sphincter present between oviduct and uterus. Vulva transverse slit with par refringens vaginae sclerotized in 2 pieces. Vagina surrounded by a well-developed constrictor muscle, extending inwards about one third of corresponding body width deep. Rectum straight, thick-walled and muscular, almost equal to anal body width long. Tail 247-295 m long, gradually tapering, posteriorly strongly ventrally curved to coiled with acutely rounded terminus. Caudal gland and spinneret absent.

Table 1. Morphometric data of Iotonchus arcuatus Khan, Araki \& Bilgrami, 2000

\begin{tabular}{lcc}
\hline & \multicolumn{2}{c}{ Iotonchus arcuatus } \\
\cline { 2 - 3 } Reference & $\begin{array}{c}\text { Hinokuma mountain-Saga } \\
\text { Prefecture (Japan) }\end{array}$ & Bidoup-Nui Ba (Vietnam) \\
\cline { 2 - 3 } & Khan et al., 2000 & Present paper \\
\hline $\mathrm{n}$ & $7+9$ & 18 + \\
$\mathrm{L}(\mathrm{mm})$ & $1.7-1.8$ & $1.3-1.6$ \\
$\mathrm{a}$ & $35-41$ & $27-33$ \\
$\mathrm{~b}$ & $4.4-4.7$ & $4.6-5.4$ \\
$\mathrm{c}$ & $4.9-6.0$ & $4.7-6.8$ \\
$\mathrm{c}$ & $9.2-10.5$ & $7.3-11$ \\
$\mathrm{~V}(\%)$ & $55-57$ & $51-55$ \\
Buccal cavity length $(\mathrm{m})$ & $38-41$ & $27-34$ \\
Buccal cavity width $(\mathrm{m})$ & $24-28$ & $15-22$ \\
Neck length (m) & $367-403$ & $279-320$ \\
Body diameter at vulva (m) & $43-48.5$ & $48-48.5$ \\
Anal body diameter (m) & $31-35$ & $28-34$ \\
Tail length (m) & $286-360$ & $247-295$ \\
\hline
\end{tabular}
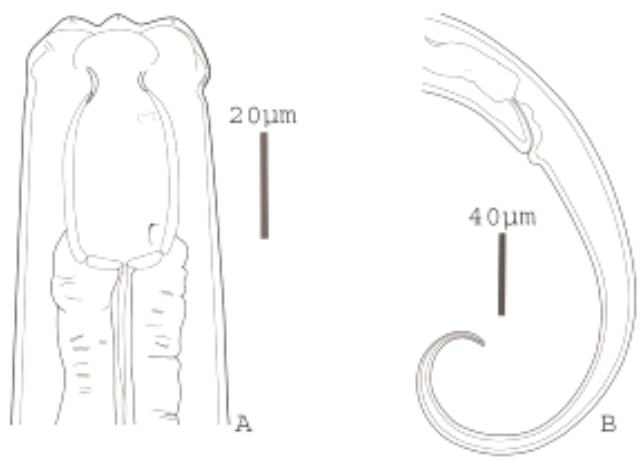

Figure 1. Iotonchus arcuatus Khan, Araki \& Bilgrami, 2000

A. Head region; B. View of female tail region.

Male: Not found.

Remark: The measurements and description of Vietnamese specimens correspond well to type population from Japan 5 exception slightly stout body $(\mathrm{a}=27-33$ vs 35-41) and smaller size of buccal cavity $(38-41 \times 24-28 \mu \mathrm{m}$ vs $27-34 \times$ 15-22 $\mu \mathrm{m})$.

Locality: Bidoup-Nui Ba National Park (Lam Dong).

\section{Iotonchus candelabri Yeates, 1992 (Fig. 2)}

Material examined: one female and one male in good condition. Measurement: see table 2.

Female: Body gently curved ventrally when fixed by heat and TAF. Cuticle smooth. Lip region width $47 \mathrm{~m}$, slightly offset from the body contour. Buccal cavity about one and half time as long as wide, with broad base, $47 \mathrm{~m}$ length and $35 \mathrm{~m}$ width. Dorsal tooth located near the base of buccal cavity with sharply apex directed forwards about $28-30 \%$ of buccal cavity length from its base. Oesophagous cylindrical, surrounds base of buccal cavity. Oesophagointestinal junction tuberculate. Secretoryexcretory system and pore not observed. Productive system didelphic-amphidelphic, both 
branches equally developed with ovary reflexed. Sphincter present between oviduct and uterus. Vulva transverse slit with par refringens vaginae sclerotized in 2 small dotes in optical section. Vagina surrounded by a well-developed constrictor muscle, extending inwards about halfway of corresponding body width deep.
Advulva papillae present, which do not protrude from body contour. Prerectum not differentiated. Rectum straight, thick-walled and muscular, less than an anal body width long. Tail elongated conoid, $280 \mathrm{~m}$ long, rounded terminus. Three caudal glands well developed. Spinneret present sub-ventrally.

Table 2. Morphometric data of Iotonchus candelabri Yeates, 1992

\begin{tabular}{|c|c|c|c|c|}
\hline \multirow[b]{3}{*}{ Reference } & \multicolumn{4}{|c|}{ Iotonchus candelabri } \\
\hline & \multirow{2}{*}{\multicolumn{2}{|c|}{$\begin{array}{c}\text { Champ de Bataille (New } \\
\text { Caledonia) } \\
\text { Yeates, } 1992\end{array}$}} & \multicolumn{2}{|c|}{ Cat Ba (Vietnam) } \\
\hline & & & & per \\
\hline $\mathrm{n}$ & 6 우 & $4 \sigma^{\lambda}$ & 1 ㅇ & $1 \delta^{\lambda}$ \\
\hline $\mathrm{L}(\mathrm{mm})$ & $2.4-2.9$ & $2.6-2.8$ & 2.5 & 2.5 \\
\hline $\mathrm{a}$ & $38-42$ & $38-43$ & 33.7 & 31 \\
\hline $\mathrm{b}$ & $4.2-4.6$ & $4.3-4.5$ & 5.2 & 4.5 \\
\hline $\mathrm{C}$ & $6.4-7.5$ & $7.1-8.6$ & 8.4 & 8.7 \\
\hline $\mathrm{C}^{\prime}$ & $7.5-9.2$ & $5.2-6.6$ & 5.5 & 4 \\
\hline $\mathrm{V} \%$ & $61-63$ & - & 58 & - \\
\hline Buccal cavity length (m) & $49-52$ & $43-48$ & 46.6 & 44 \\
\hline Buccal cavity width (m) & $35-41$ & $33-38$ & 35.3 & 34 \\
\hline Neck length (m) & ? & ? & 463.6 & 436.3 \\
\hline Body diameter at vulva (m) & ? & ? & 74.3 & 81 \\
\hline Anal body diameter (m) & ? & ? & 51 & 57.5 \\
\hline Tail length (m) & $350-450$ & $310-400$ & 279 & 230 \\
\hline Spicules length (m) & - & $77-83$ & - & 90 \\
\hline Supplements & - & $12-16$ & - & 14 \\
\hline
\end{tabular}

?. no information.
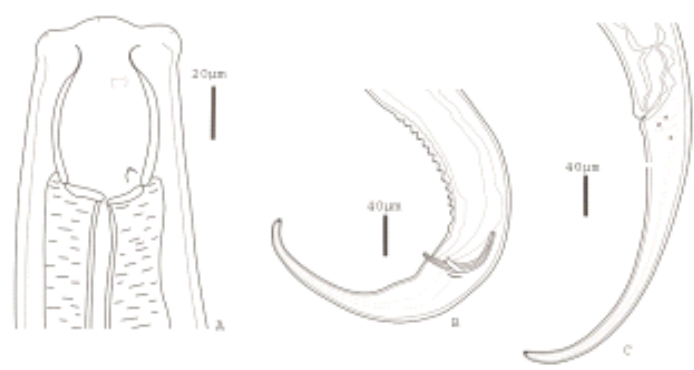

Figure 2. Iotonchus candelabri Yeates, 1992

A. Head region; B. View of male tail reagion; C. View of female tail region.

Male: Similar to female in general morphology. Testes paired, opposed, outstretched. Prerectum distinct, beginning slightly anterior to copulatory muscules. Midventral supplements 14 with a single ventral pore about body width anterior to first. Spicules paired, arcuate, with little cephalic expansion. Lateral guiding pieces $16 \mu \mathrm{m}$ and gubernaculum $12.5 \mu \mathrm{m}$, well developed. Tail elongate conoid, terminus rounded. Three caudal glands well developed, spinneret present sub-ventral terminus.

Remark: The measurements of Vietnamese specimens fitted well with type population from New Caledonia [15] exception slightly stout body ( $\mathrm{a}=31-34$ vs 38-43) and longer spicules.

Locality: Cat Ba National Park (Hai Phong).

Iotonchus paracutus Vinciguerra \& Orselli, 2000 (Fig. 3)

Material examined: one female in good condition. Measurement: see table 3.

Female: Body large and robust, tapering at the posterior extremity. Body width at cardia 
1.4 lip region width. Cuticle finely transversely striated, $2 \mathrm{~m}$ thick at the mid-body. Amphidial aperture small, cup shaped, beginning at $21 \mu \mathrm{m}$ from anterior end of body. Lip region broader than adjoining body and set off by constriction. Buccal cavity heavily sclerotized, $40.5 \mu \mathrm{m}$ length and $31.5 \mu \mathrm{m}$ width. Dorsal tooth located almost at its base with sharply apex directing forwards, located at $20 \%$ buccal cavity length from its base. Oesophagous cylindrical, surrounds base of buccal cavity. Nerve ring located at $52 \%$ of the pharyngeal length from anterior end. Oesophago-intestinal junction tuberculate. Productive system didelphicamphidelphic, both branches equally developed with ovary reflexed. Vulva transverse slit with par refringens vaginae sclerotized in 2 pieces. Prerectum not differentiated.

Rectum straight, thick-walled and muscular, about 0.7 anal body width long. Tail elongated conoid, straight, $172 \mathrm{~m}$ long or about 4 anal body diameter long, rounded terminus. Spinneret absent.

Table 3. Morphometric data of Iotonchus paracutus Vinciguerra \& Orselli, 2000

\begin{tabular}{lccc}
\hline \multirow{2}{*}{ Reference } & \multirow{2}{*}{ Distribution } & \multicolumn{3}{c}{ Iotonchus paracutus } \\
\cline { 2 - 4 } & \multicolumn{2}{c}{ Siracusa (Italia) } & Cat Ba (Vietnam) \\
\cline { 2 - 4 } & \multicolumn{2}{c}{ Vinciguerra \& Orselli, 2000 } & Present paper \\
\hline $\mathrm{n}$ & 2 q & 2 O & 1 q \\
$\mathrm{L}(\mathrm{mm})$ & $1.9-2.2$ & 1.6 & 1.82 \\
$\mathrm{a}$ & $23-26$ & $23-24$ & 20 \\
$\mathrm{~b}$ & $5-5.1$ & 4.6 & 4.5 \\
$\mathrm{c}$ & $9.4-10.9$ & $10.5-11.3$ & 10.5 \\
$\mathrm{c}$ & $3.5-4.6$ & $2.8-3$ & 3.8 \\
V \% & $62-63$ & - & 62 \\
Buccal cavity length (m) & $42^{*}$ & $42.5 *$ & 40.5 \\
Buccal cavity width (m) & $35-40$ & $30-32.5$ & 31.5 \\
Neck length (m) & $370-430$ & $345-347$ & 400 \\
Body diameter at vulva (m) & 82.5 & $65-70$ & 91 \\
Anal body diameter (m) & $47.5-50$ & 50 & 45.5 \\
Tail length (m) & $170-230$ & $140-150$ & 172.7 \\
Spicules length (m) & - & $57.5-62.5$ & - \\
Supplements & - & $10-11$ & - \\
\hline * The & & &
\end{tabular}

* The measurement was calculated from original figure.

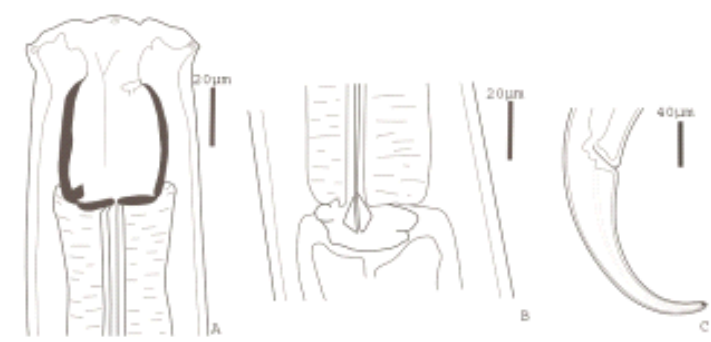

Figure 3. Iotonchus paracutus Vinciguerra \& Orselli, 2000

A. Buccal cavity; B. Oesophago - intestinal junction region; $\mathrm{C}$. View of female tail region.

Male: Not found in Vietnam.
Remark: The measurements and description of Vietnamese specimen correspond well to type population from Italia 14.

Locality: Cat Ba National Park (Hai Phong).

\section{Iotonchulus pusillus Loof, 2006 (Fig. 4)}

Material examined: 11 females in good condition. Measurements: see table 4.

Female: Moderately slender nematodes of small size, 0.7-0.9 mm. Body curved ventrally behind neck and more strongly towards posterior end of body. Cuticle smooth and about $1 \mathrm{~m}$ thick. Lateral chord occupying ca one quarter body diameter. Lip region rounded, 
offset slightly with the body adjacent. Amphidial fovea cup shaped, aperture $3 \mathrm{~m}$ beginning at the anterior end of buccal cavity. Buccal cavity elongated oval with 21-23 m long and 11.5-16 m width. Dorsal tooth with sharply apex directing forwards located 20-25\% buccal cavity length from its base. Cardia between oesophagous and intestine tuberculate.
Reproductive system mono-prodelphic, anterior branch well developed with ovary reflexed. Vulva transverse slit with par refringens vaginae un-cleared. Rectum straight, thickwalled and muscular, almost equal to anal body width long. Tail 110-148 m long, tail curved more strongly terminally to form a spiral. Caudal gland and spinneret not seen.

Table 4. Morphometric data of Iotonchus pusillus Loof, 2006

\begin{tabular}{|c|c|c|c|}
\hline \multirow{3}{*}{ Reference } & \multicolumn{3}{|c|}{ Iotonchus pusillus } \\
\hline & $\begin{array}{c}\text { Hutan Phuchong } \\
\text { (Malaysia) }\end{array}$ & $\begin{array}{c}\text { Pulau Pinang } \\
\text { (Malaysia) }\end{array}$ & $\begin{array}{c}\text { Bidoup - Nui Ba } \\
\text { (Vietnam) }\end{array}$ \\
\hline & \multicolumn{2}{|c|}{ Loof, 2006} & Present paper \\
\hline $\mathrm{n}$ & 10 운 & 3 ㅇ & 11 운 \\
\hline $\mathrm{L}(\mathrm{mm})$ & $0.7-0.83$ & $0.91-0.96$ & $0.75-0.9$ \\
\hline $\mathrm{a}$ & $24-28$ & $29-30$ & $22-33$ \\
\hline b & $3.6-4.0$ & $4.1-4.3$ & $3.9-4.6$ \\
\hline $\mathrm{c}$ & $7.2-8.1$ & $7.0-7.9$ & $5.1-6.8$ \\
\hline$c^{\prime}$ & 4.7- 5.9 & $5.8-6.9$ & $5.5-7.5$ \\
\hline $\mathrm{V} \%$ & $64-67$ & $63-65$ & $59-64$ \\
\hline Buccal cavity length (m) & $22-24$ & $23-24$ & $20.5-23$ \\
\hline Buccal cavity width (m) & $10-12$ & $11-13$ & $11.5-14$ \\
\hline Neck length (m) & $193-215$ & $222-229$ & $180-211$ \\
\hline Body diameter at vulva (m) & $29-33$ & $32-33$ & $22.5-36$ \\
\hline Anal body diameter (m) & $19-21$ & $19-21$ & $18-27$ \\
\hline Tail length (m) & 94-114 & $121-128$ & $110-148$ \\
\hline
\end{tabular}
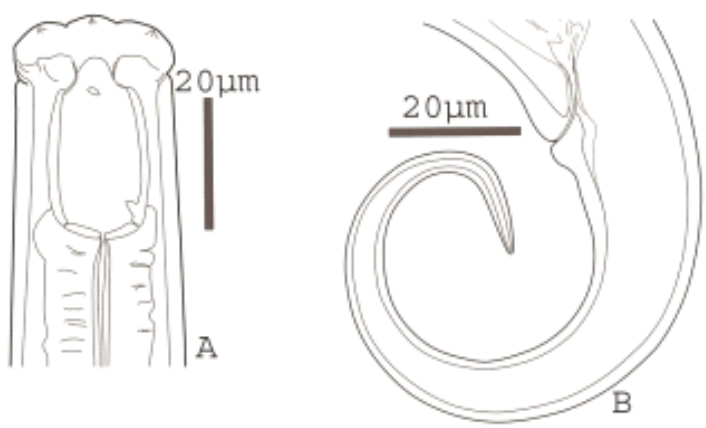

Figure 4. Iotonchus pusillus Loof, 2006

A. Head region; B. View of female tail region.

Male: Not found.

Remark: The above description and measurements fitted well to type population from Malaysia 6.

Locality: Bidoup - Nui Ba National Park (Lam Dong).

\section{Iotonchus \\ singaporensis \\ Ahmad, Baniyamuddin and Jairajpuri, 2006 (Fig. 5)}

Examined specimens: 4 females in good condition. Measurements: see table 5.

Female: Moderately slender nematodes of medium size, 1.2-1.3 mm long. Habitus after fixation ventrally curved, tapering gradually towards posterior region and terminating into a long, ventrally arcuate tail. Cuticle smooth, 2-3 $\mu \mathrm{m}$ thick at the mid-body and 3-4 $\mu \mathrm{m}$ on tail. Lateral chords one-fourth to one-third of body width at the mid-body. Lip region clearly demarcated, distinctly wider than adjoining body. Labial pappilae slightly projected above labial contour. Amphids cup shaped with slit like apertures near base of lips. Buccal cavity medium size, barrel shaped, flat at base, strongly sclerotized, 30-32 $\mathrm{m}$ long and 17-18 m wide. Dorsal tooth small, anteriorly directed, its tip at about $23-28 \%$ from the base. Nerve ring at 38- 
$40 \%$ neck length from anterior end. Excretory pore distinct. Oesophago-intestinal junction tuberculate. Reproductive system monoprodelphic, anterior branch well developed with ovary reflexed, posterior branch completely absent. Vulva transverse slit, vagina anteriorly directed, par refringens vaginae sclerotized as 2 triangular pieces. Rectum straight, thick-walled and muscular, 0.5-0.6 anal body width long. Tail elongate, ventrally arcuate, gradually tapering to an almost acute terminus, $270-280 \mathrm{~m}$ long or 810 anal body width long. Caudal glands weakly developed, grouped and spinneret presents subventral terminus.

Table 5. Morphometric data of Iotonchus singaporensis Ahmad, Baniyamuddin and Jairajpuri, 2006

\begin{tabular}{|c|c|c|c|}
\hline \multirow{3}{*}{ Reference } & \multirow{3}{*}{ Distribution } & \multicolumn{2}{|c|}{ Iotonchus singaporensis } \\
\hline & & $\begin{array}{l}\text { Kent Ridge park } \\
\text { (Singapore) }\end{array}$ & $\begin{array}{c}\text { Cat Ba park } \\
\text { (Vietnam) }\end{array}$ \\
\hline & & Ahmad et al., 2005 & Present paper \\
\hline $\mathrm{n}$ & & 15 운 & 4 q \\
\hline $\mathrm{L}(\mathrm{mm})$ & & $1.039-1.232$ & $1.2-1.3$ \\
\hline $\mathrm{a}$ & & $26-31$ & $26-27$ \\
\hline $\mathrm{b}$ & & $3.7-4.2$ & $3.8-4.1$ \\
\hline $\mathrm{c}$ & & $5.1-6.7$ & 4.6-4.9 \\
\hline $\mathrm{c}^{\prime}$ & & $6.3-8.3$ & $8-9.4$ \\
\hline $\mathrm{V} \%$ & & $59-65$ & $60-62$ \\
\hline Buccal cavity length $(\mu \mathrm{m})$ & & $31-33$ & $30-32$ \\
\hline Buccal cavity width $(\mu \mathrm{m})$ & & $18-19$ & $16.8-17.6$ \\
\hline Body diameter at vulva $(\mu \mathrm{m})$ & & $36-41$ & $34-54$ \\
\hline Anal body diameter $(\mu \mathrm{m})$ & & $22-25$ & $28-33$ \\
\hline Neck length $(\mu \mathrm{m})$ & & $262-296$ & $290-333$ \\
\hline Tail length $(\mu \mathrm{m})$ & & $164-225$ & $270-276$ \\
\hline
\end{tabular}
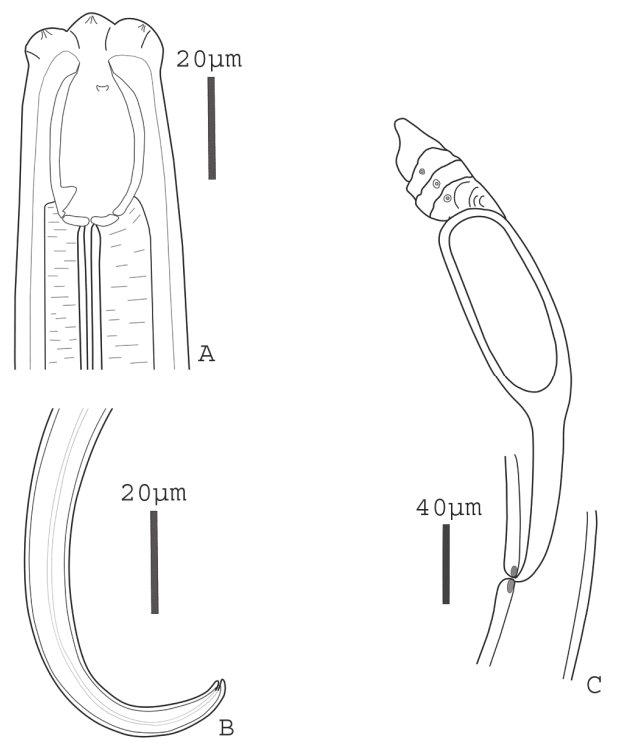

Figure 5. Iotonchus singaporensis Ahmad, Baniyamuddin and Jairajpuri, 2006.

A. Head region; B. Female reproductive region; C. Tail terminus.
Male: Not found.

Remark: The above description and measurements fitted well to type population from Singapore 1.

Locality: Cat Ba National Park (Hai Phong).

Discussion about Iotonchus thui Nguyen, 2006 and I. helicus Nguyen, 2006

Note for history of Iotonchus thui Nguyen, 2006

In 2005, "Iotonchus thui" was first recorded by Nguyen $\mathrm{Vu}$ Thanh as "Iotonchus thui Coomans \& Nguyen Thi Thu, 2001" in the book: Predatory Nematodes of Mononchida in Vietnam [8], based on results of the master thesis of Nguyen Thi Thu (2001, Ghent University, Belgium) [7]. In this thesis, the new species was described and illustrated but remained unnamed. It was based on material with males and females from two different localities. Moreover, the thesis is not considered 
a publication. Therefore, the species name is a nomen nudum.

In 2006, "Iotonchus thui" was described and published as a new species again by Nguyen $\mathrm{Vu}$ Thanh in Tap chi Sinh hoc (Journal of Biology) [9] though with the wrong illustration i.e. the illustration of $I$. nayari Mohandas \& Prabhoo, 1979 in the master thesis was used [7]. The female holotype and paratypes were recorded from $\mathrm{Ha}$ Giang province while the male paratypes were from Cuc Phuong National Park, Ninh Binh province; no detailed information was provided on the type locality. According to article 16.4.1 of ICZN [16], the type series should come from the same locality. One should be very careful when combining specimens from different localities.

Further, the author explained that the name "thui" was referring to the person firstly collected specimens. However, according to article 31.1 .2 of ICZN [16], as indeed this was the real new species "thui" should have been "thuae" since the person is a female.

For all the above mentioned reasons, I. thui can be considered a sp.inq.

\section{For the species of Iotonchus helicus Nguyen, 2006}

The I. helicus Nguyen, 2006 was published in the same article with $I$. thui species [9]. However, the illustration does not fit with its measurements considering the given scale bar. Therefore, we decided to check again holotype and paratypes of this species, which were deposited and preserved at the Department of Nematology, IEBR (Dr. Nguyen Vu Thanh's collection). Unfortunately, all type material including holotype and paratypes were not available. His collection still has four slides with bad quality. On each of these slides, "Buon Ma Thuat" corresponds on the left hand and "Iotonchus sp." on the right hand. In my opinion, this information is not enough evidences to demonstrate that these slides are syntypes of I. helicus species.

On original description, I. helicus was characterized with small body size $(\mathrm{L}=1.17$ 1.66); short tail; $\mathrm{a}=16-21 ; \mathrm{c}=5.5-6.9$; spinneret sub-terminal, ventral terminus [9]. However, the author has compared I. helicus with $I$. rayongensis Buangsuwon \& Jenssen, 1966, I. thailandensis Buangsuwon \& Jenssen, 1966 (was synonym of I. transkeiensis Heyns \& Lagerway, 1965) and I. basidontus Clark, 1961 without any similarity of main characters. I. helicus has spinneret on sub-ventral terminus while I. rayongensis or I. basidontus has spinneret on dorsal terminus or terminal and without spinneret in case species of I. transkeiensis [2].

I. helicus and I. indicus Jairajpuri, 1969 are very similar in main diagnostic characters as: (i) female didelphic; (ii) spinneret sub-ventral; (iii) small species $\mathrm{L}=1.2-2 \mathrm{~mm}$; (iv) advulvar body pores absent and (v) as well as in measurements (table 6) $[3,4,6,10]$; all the measurements of I. helicus fit well those of I. indicus from original population in Indian as well as other populations from El Salvador, Malaysia and Vietnam exception slightly stout body ( see "a" and 'c'" ratios) $[3,4,6,10]$.

For all the above mentioned reasons, I. helicus can be considered a junior synonym of I. indicus Jairajpuri, 1969.

Table 6. Comparison of main morphometric data between I. helicus Nguyen, 2006 and I. indicus Jairajpuri, 1969

\begin{tabular}{|c|c|c|c|c|c|c|}
\hline \multirow[b]{3}{*}{ Reference } & \multirow{3}{*}{ Locality } & Iotonchus & \multicolumn{4}{|c|}{ Iotonchus indicus } \\
\hline & & Vietnam & India & El Salvador & Malaysia & Vietnam \\
\hline & & $\begin{array}{l}\text { Nguyen, } \\
2006\end{array}$ & $\begin{array}{c}\text { Jairajpuri, } \\
1969\end{array}$ & $\begin{array}{c}\text { Baqri \& } \\
\text { Jairajpuri, } \\
1973\end{array}$ & $\begin{array}{l}\text { Loof, } \\
2006\end{array}$ & $\begin{array}{c}\text { Nguyen, } \\
2007\end{array}$ \\
\hline $\mathrm{L} \mathrm{mm}$ & & $1.17-1.66$ & $1.54-1.97$ & $1.23-1.79$ & $1.93-2$ & $1.47-1.75$ \\
\hline
\end{tabular}


Vu Thi Thanh Tam

\begin{tabular}{lccccc}
\hline $\mathrm{V} \%$ & $52-57$ & $57-65$ & $56-69$ & $55-57$ & $53.6-58.5$ \\
$\mathrm{a}$ & $16-21$ & $21-32$ & $25-28$ & $32-35$ & $22.7-27.5$ \\
$\mathrm{~b}$ & $3.7-4.6$ & $4-4.8$ & $3.7-4.1$ & 4.7 & $4-4.5$ \\
$\mathrm{c}$ & $5.5-6.9$ & $5-8$ & $5-6$ & $5-5.2$ & $4.8-5.4$ \\
c' & $4.3-6.1$ & $6-9$ & $?$ & $10.2-10.4$ & $7.6-9$ \\
\hline $\begin{array}{l}\text { Buccal cavity length } \\
(\mu \mathrm{m})\end{array}$ & $36-45$ & $40-47$ & $42-50$ & $43-45$ & $44-50.6$ \\
\hline $\begin{array}{l}\text { Buccal cavity width } \\
(\mu \mathrm{m})\end{array}$ & $19.8-27.9$ & $28-32$ & $26-29$ & $23-24$ & $23.9-25.8$ \\
\hline Tail length $(\mu \mathrm{m})$ & $178-260$ & $200-360$ & $218-345$ & $383-388$ & $286-342$ \\
\hline ?: no information. & & & & &
\end{tabular}

In current paper, 18 species of the genus Iotonchus have been recorded in Vietnam. The following adapted key to species is based on Ahmad and Jairajpuri (2010):

1a) Female genital organ mono-prodelphic, post-uterine sac very short or absent...............2

1b) Female genital organ didelphic. Tail conoid-arcuate or filiform, shorter than 25 anal body

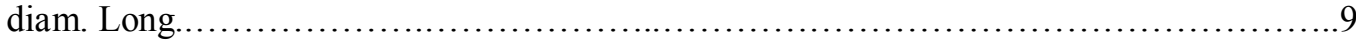

2a) Post uterine sac shorter than 1 anal body diameter long........................ paratrichurus

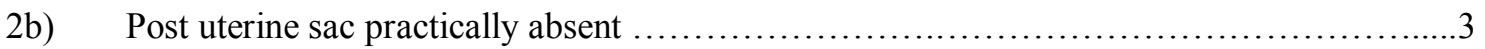

3a) Tail shorter than 4 anal body diam. long ................................. Inisostomus

3b) Tail longer than 6 anal body diam. long ................................................... 4

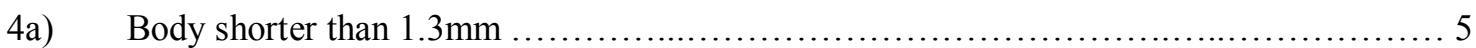

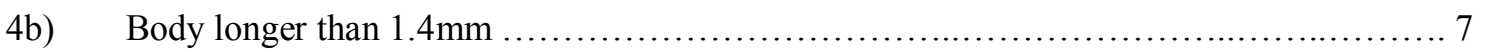

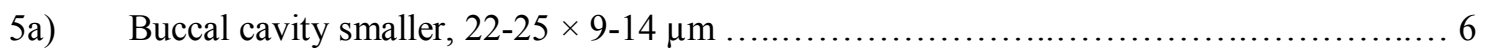

5b) Buccal cavity larger, 31-36 $\times 18-20 \mu \mathrm{m} \ldots \ldots \ldots \ldots \ldots \ldots \ldots \ldots \ldots \ldots \ldots \ldots \ldots \ldots \ldots$. singaporensis

6a) Tail terminus sub-acute, spinneret present ............................. I. chantaburensis

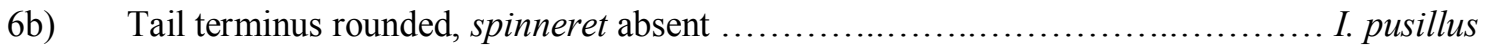

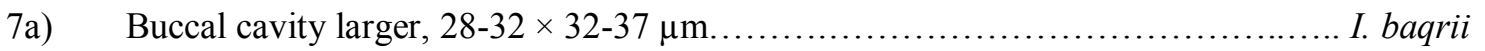

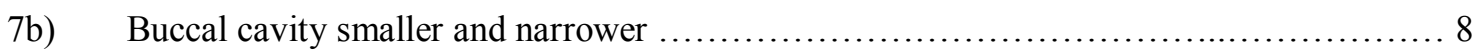

8a) Tail shorter than 12 anal body diam. long ...................................... I. silvallus

8b) Tail longer than 15 anal body diam. long ...................................... Irichurus

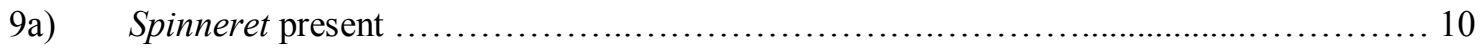

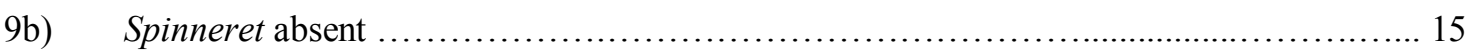

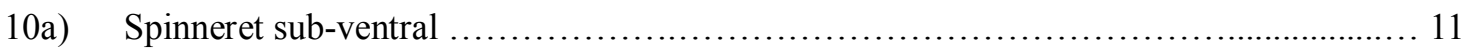

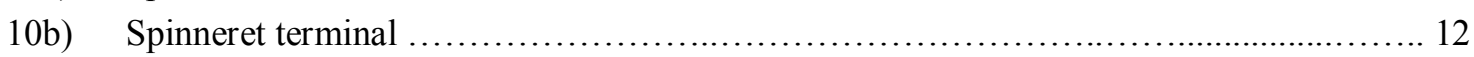

11a) Small species, $\mathrm{L}=1.2-2 \mathrm{~mm}$. Advulvar papillae absent ............................... I. indicus

11b) Species not as small, $\mathrm{L}=2.2-3.4 \mathrm{~mm}$. Advulvar papillae present .............. I. candelabri

12a) Body longer than $3 \mathrm{~mm}$................................................ risoceiae

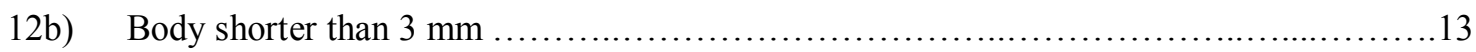

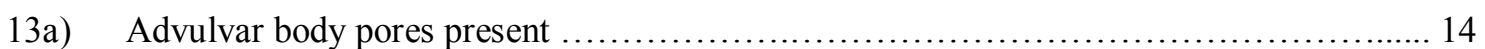

13b) Advulvar body pores absent .............................................. I. basidontus

14a) Buccal cavity narrowing posteriorly. Tail shorter, ca 6-9 anal body diam.

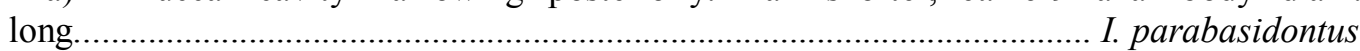


14b) Buccal cavity not narrowing posteriorly. Tail longer, ca 4-6 anal body diam. Long..I. nayari

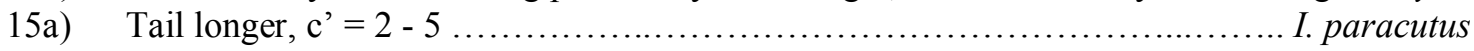

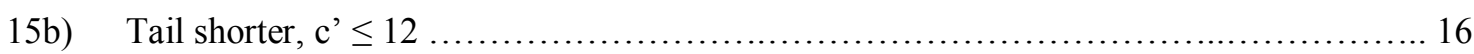

16a) Lateral organs present ........................................... I. transkeiensis

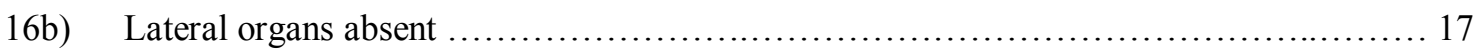

17a) Tail strongly curved, clearly narrowing near terminus ........................ I. arcuatus

17b) Tail only slightly ventrally curved with thicker terminal part..................... I. clarki

Acknowledgments: The author would like to thank Prof. Dr. Junichi Kojima, Commissioner of International Commission on Zoological Nomenclature and Prof. Dr. Wilfrida Decraemer at University of Ghent, Belgium for good advice in discussion.

\section{REFERENCES}

1. Ahmad W., Baniyamuddin M., Jairajpuri M. S., 2005. Three new and known species of Mononchida (Nematoda) from Singapore. Journal of Nematode Morphology and Systematics, 7(2): 97-107.

2. Ahmad W., Jairajpuri M. S., 2010. Mononchida: The predaceous Nematodes. Brill Leiden-Boston. 288pp.

3. Baqri S. Z., Jairajpuri M. S., 1973. Studies on Mononchida. V. The mononchd of El Salvador with descriptions of two new genera, Actus and Paracrassibucca. Nematologica, 19: 326-333.

4. Jarajpuri M. S., 1969. Studies on Mononchida of India. I. The genera Hadronchus, Iotonchus and Miconchus and a revised classification of Mononchida, new order. Nematologica, 15: 557-581.

5. Khan Z., Araki M., Bilgrami A. L., 2000. Iotonchus sagaensis sp.n., I. arcuatus sp.n. and Miconchus japonicus sp.n. (Nematoda: Mononchida) from Japan. International Journal of Nematology, 10(2): 143-152.

6. Loof P. A. A., 2006. Mononchina (Dorylaimida) from Western Malaysia. Nematology, 8(2): 287-310.

7. Nguyen Thi Thu, 2001. A taxonomic study of Mononchida from Vietnam. Master thesis Ghent University, Belgium, 48 pp.

8. Nguyen $\mathrm{Vu}$ Thanh, 2005. Predaceous nematodes of Mononchida in Vietnam. Agriculture publishing house. 240pp.

9. Nguyen $\mathrm{Vu}$ Thanh, 2006. Two new predatory nematode species Iotonchus helicus sp. nov and Iotonchus thui sp. nov (nematoda: Mononchida) from Vietnam. Tap chi Sinh hoc, 28(2): 10-16.

10. Nguyen Vu Thanh, 2007. Fauna of Vietnam. Vol. 22. Free living nematodes: Mononhysterida, Araeolaimida, Chromadorida, Rhabditida, Enoplida, Mononchida, Dorylaimida. Science and Technics Publishing House. 455pp.

11. Seinhorst J. W., 1959. A rapid method for the transfer of nematodes from fixative to anhydrous glycerin. Nematology, 4: 67-69.

12. Southey J. F., 1986. Laboratory methods for work with plant and soil nematodes. London: Her Majesty' Stationery Office. 202pp.

13. Siddiqi M. R., 2001. Study of Iotonchus species (Mononchida) from West Africa with descriptions of eleven new species. International Journal of Nematology, 11(1): 104-123.

14. Vinciguerra M. T., Orselli L., 2000. Nematodes from Italian sand dunes. 5. Two new species of Dorylaimida and three known species of Enoplida. Nematol.medit., 28: 301-312.

15. Yeates G. W., 1992. Nematodes from New Caledonia. 1. Introduction and Mononchoidea. Fundam. appl. Nematol., 15(2): 101-126.

16. Website: Www.nhm.ac.uk/hostedsites/iczn/code/. The website of International Code of Zoological Nomenclature online. 


\title{
GHI NHẬN MỚI 5 LOÀI THUỘC GIỐNG IOTONCHUS (MONONCHIDA, IOTONCHIDAE) CHO KHU HỆ TUYẾN TRÙNG VIÊTT NAM VỚI KHÓA ĐỊNH LOẠI CÁC LOÀI CỦA GIỐNG NÀY Ở VIẸT NAM
}

\author{
Vũ Thị Thanh Tâm
}

Viện Sinh thái và Tài nguyên sinh vật, Viện Hàn lâm KH \& CN Việt Nam

\section{TÓM TÁTT}

Tuyến trùng sống tự do trong đất thuộc bộ ăn thịt Mononchida đã được nghiên cứu ở Việt Nam từ những năm 90 của thế kỷ trước và đến nay đã ghi nhận được 56 loài thuộc 4 họ 10 giống tại các địa điểm nghiên cứu trên cả nước. Cho đến nay, đã ghi nhận được 15 loài thuộc giống Iotonchus (họ Iotonchidae) ở Việt Nam.

Trong bài báo này, 5 loài tuyến trùng thuộc giống Iotonchus: I. arcuatus, I. candelabri, I. paracutus, I. pusillus và $I$. singaporensis được ghi nhận lần đầu tiên cho khu hệ tuyến trùng Việt Nam, những mẫu của 5 loài này thu được từ hai vườn quốc gia Cát Bà và Bidoup-Núi Bà.

Hai loài tuyến trùng đã được ghi nhận ở Việt Nam trước đây là $I$. thui Nguyen, 2006 và $I$. helicus Nguyen, 2006 được xem là đồng vật mới. Khóa định loại cho tất cả các loài thuộc giống Iotonchus đã được xây dựng. Như vậy, cho đến nay, có 18 loài tuyến trùng thuộc giống Iotonchus đã được ghi nhận cho khu hệ tuyến trùng Việt Nam.

Tù khóa: Mononchida, Iotonchidae, Iotonchus, taxonomy.

Ngày nhận bài: 25-5-2015 